\title{
Initial Experience with p64: A Novel Mechanically Detachable Flow Diverter for the Treatment of Intracranial Saccular Sidewall Aneurysms
}

\author{
S. Fischer, M. Aguilar-Pérez, E. Henkes, W. Kurre, O. Ganslandt, H. Bäzner, and H. Henkes
}

\begin{abstract}
BACKGROUND AND PURPOSE: Flow diverters are important tools for the treatment of intracranial aneurysms. We report a retrospective evaluation of the safety and efficacy of p64, a fully resheathable, detachable flow diverter, in the endovascular treatment of intracranial sidewall aneurysms.
\end{abstract}

\begin{abstract}
MATERIALS AND METHODS: Results of 121 patients with 130 aneurysms (median neck size, $3 \mathrm{~mm}$; median fundus size, $4 \mathrm{~mm}$ ), treated from April 2012 through October 2014, were analyzed. Aneurysms were unruptured or beyond the acute SAH phase. Thirteen aneurysms were located in the posterior circulation. Twenty-three aneurysms had previous saccular treatment but no previous parent vessel stent placement. In 19 aneurysms, a combination of coiling and flow diversion was performed.

RESULTS: Successful p64 deployment was achieved in 127/130 aneurysms. The average number of p64s used was 1.1 per aneurysm. The rates of transient and permanent morbidity and mortality were $5 \%, 1.7 \%$, and $0.8 \%$, respectively. Three-month DSA follow-up in $123 / 130$ aneurysms showed complete occlusion in 58.5\%. Nine-month DSA follow-up in 93/106 (87.7\%) eligible aneurysms showed complete occlusion in $79.6 \%$. Late follow-up (median, 496 days) has already been performed in 35 aneurysms, showing complete occlusion in 30 (85.7\%).

CONCLUSIONS: p64 offers an efficacious treatment option for intracranial sidewall aneurysms with a high aneurysm occlusion and an acceptable complication rate. The possibility of repositioning or removing the device was an advantage. The higher attenuation may lead to fewer devices per case and early aneurysm occlusion. Long-term follow-up data are pending.
\end{abstract}

ABBREVIATIONS: $L L=$ lumen loss; $P E D$ = Pipeline Embolization Device

$F_{\text {tis }}$ ow diversion has become an accepted endovascular treatment for selected intracranial aneurysms. Both the clinical and endovascular treatment data with follow-up results of several patient cohorts treated with different flow diverters have been published during the past 5 years. ${ }^{1-6}$ In Europe, 6 flow diverters are currently available for clinical use (Silk+, Balt Extrusion, Mont-

Received November 13, 2014; accepted after revision March 17, 2015.

From the Neuroradiologische Klinik (S.F., M.A.-P., E.H., W.K., H.H.), Neurochirurgische Klinik (O.G.), and Neurologische Klinik (H.B.), Neurozentrum, Klinikum Stuttgart, Stuttgart, Germany; and the Institut für Diagnostische und Interventionelle Radiologie, Neuroradiologie und Nuklearmedizin (S.F.), Universitätsklinikum Knappschaftskrankenhaus, Bochum, Germany.

Preliminary data from this series was presented at: Annual Meeting of the American Society of Neuroradiology and the Foundation of the ASNR Symposium, May 17-22, 2014; Montreal, Quebec, Canada.

The Guidelines of the Declaration of Helsinki of the World Medical Association in its current version (WMA, 2004), the Guidelines of Good Clinical Practice (CPMP/ $\mathrm{ICH} / 135 / 95)$, and demands of the national medical and data protection laws were followed.

Please address correspondence to Hans Henkes, MD, Neuroradiologische Klinik, Neurozentrum, Klinikum Stuttgart, Katharinenhospital, Kriegsbergstr 60, D-70174 Stuttgart, Germany; e-mail: hhhenkes@aol.com

http://dx.doi.org/10.3174/ajnr.A4420 morency, France; PED FLEX, Medtronic, Dublin, Ireland; FRED, MicroVention, Tustin, California; NeuroEndoGraft, Stryker Neurovascular, Fremont, California; Derivo, Acandis, Pforzheim, Germany; p64, phenox, Bochum, Germany). The principle of flow diversion is the redirection of blood flow away from the aneurysm along the longitudinal axis of the parent artery. This is accomplished by endoluminal placement of the device across the aneurysm neck. The hemodynamic effect depends mainly on the porosity of the mesh. ${ }^{7}$ The occlusion of the aneurysm or the failure to occlude and potential complications follow device-specific patterns. An understanding of the technical features and functions of different flow diverters may help proper patient selection and procedure performance.

We report our experience in the treatment of intracranial saccular sidewall aneurysms with p64, a new mechanically detachable flow diverter.

\section{MATERIALS AND METHODS p64 Flow-Modulation Device}

The p64 is a braided tubular implant consisting of 64 nitinol wires. It is available with nominal diameters from 2.5 to $5 \mathrm{~mm}$ with 0.5 


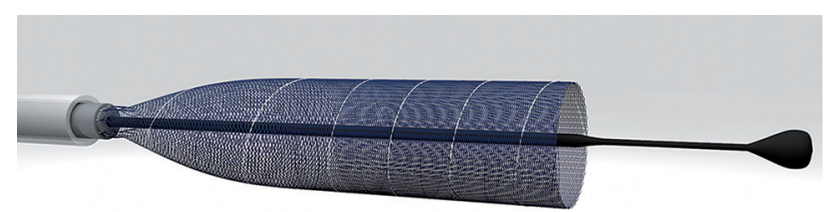

A

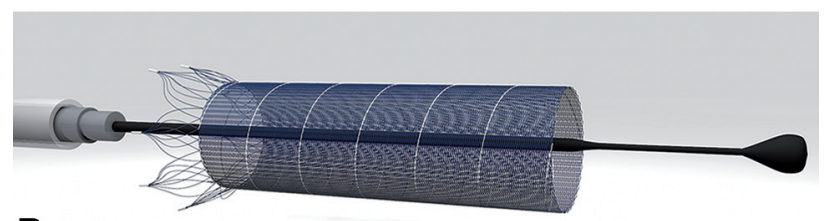

B

FIG 1. Detachment mechanism of the p64. Eight bundles, each containing 8 wires, are attached to a slotted crown $(A)$ and released from there by pulling a polymer hypotube in the proximal direction $(B)$. Image courtesy of phenox.

$\mathrm{mm}$ increments. The nominal length ranges from 9 to $30 \mathrm{~mm}$ with 3 -mm increments. Visibility under $\mathrm{x}$-ray fluoroscopy is possible by 2 platinum wires wrapped around opposing nitinol wires of the braided shaft. The 64 wires of the implant are grouped into 8 bundles proximally, each consisting of 8 individual wires. The ends of each bundle carry a radiopaque marker with a $0.5-\mathrm{mm}$ length. The 8 bundles are mounted onto a slotted crown on the distal end of a stainless steel delivery wire. A polymer tube covers the crown, securing the 8 markers in the slotted crown and extends proximally for $180 \mathrm{~cm}$ over the delivery wire (Fig 1). A torque device is used to lock the polymer tube to the delivery wire to prevent premature detachment of the implant from the slotted crown. The p64 is available with and without a distal wire. In the model with a wire, the distal end of this wire has a radiopaque marker. The distal wire increases flexibility and support during navigation and deployment of the implant. Once deployed, the p64 can be fully recovered by advancing the microcatheter while pulling back on the delivery wire.

Detachment of p64 starts with repositioning of a torque device approximately $15 \mathrm{~mm}$ proximal to the end of the hypotube (Fig $2 A,-B$ ).Pulling the hypotube toward the torque device (Fig 2C) will result in movement of this tube in the proximal direction, thereby releasing the 8 markers from the slotted crown.

The porosity (defined as the proportion of the surface area without metal coverage over the total surface area) of the implanted device depends on the implant model size and the relationship between the diameter of the device and the target vessel. Under standardized conditions (ie, in a tube with a nominal diameter), the porosity of p 64 varies from $51 \%$ to $60 \%$; the coverage (defined as the proportion of the surface area with metal coverage over the total surface area) varies from $35 \%$ to $49 \%$; and the area of an individual cell is largely dependent on the expansion status of the device. Undersizing increases and oversizing decreases the coverage. The radial force of the p64 at nominal diameters is similar to that of the PED FLEX (PED; Covidien, Irvine, California).

\section{Patient Population and Aneurysm Characteristics}

Given the high frequency of a fundus diameter of $\leq 6 \mathrm{~mm}$ in ruptured aneurysms, all treatment options are discussed and
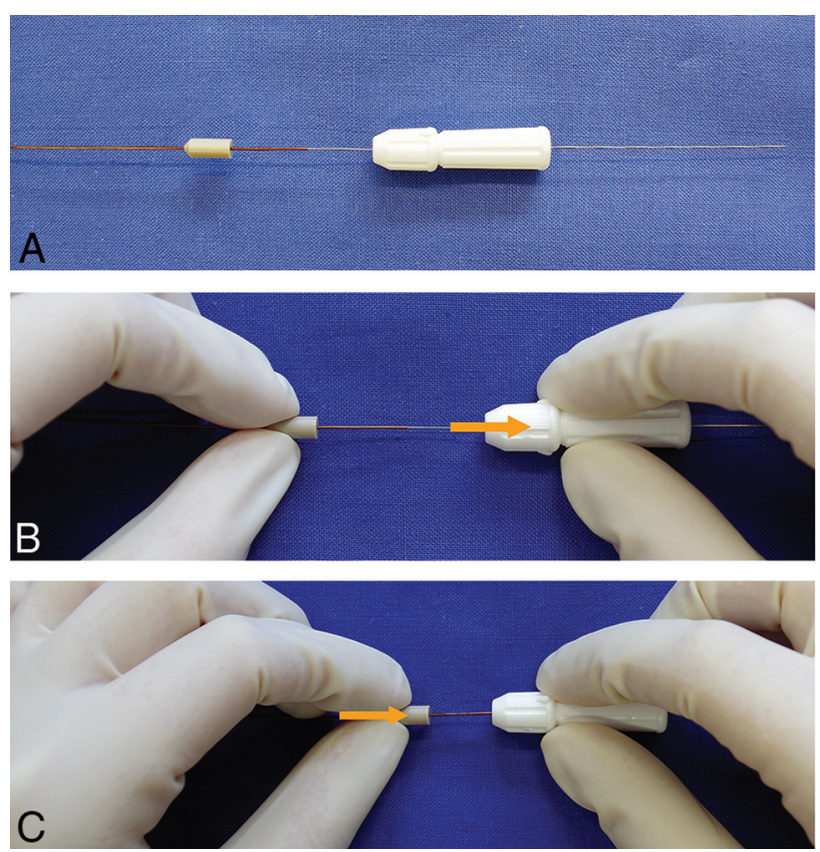

FIG 2. Manual detachment of the p64. The p64 comes with a torque device, which is locked over the hypotube to hold this hypotube in position $(A)$. The detachment starts with unlocking the torquer, repositioning it, and again locking it approximately 15 $\mathrm{mm}$ proximally $(B)$. A handle on the hypotube is then moved proximally (C).

offered to all patients with intradural aneurysms, unless individual aspects (eg, major comorbidity, older than 80 years of age, anticipated treatment difficulty, and so forth) are considered potential contraindications. A multidisciplinary neurovascular team, including vascular neurosurgeons and stroke neurologists, discussed the treatment concept for each patient in advance. All patients were informed about the nature of their disease and the intended treatment and potential alternatives, with informed consent obtained at least 24 hours before the procedure.

A retrospective review of all medical records and radiographic studies of patients treated with the p64 between April 2012 and October 15, 2014 was performed.

The selection criteria for the endovascular treatment with the p64 and inclusion in this retrospective analysis are summarized in Table 1.

According to these criteria, 121 patients ( 86 women; 35 men; median age 58 years (range, 26-79 years) with 130 intracranial aneurysms were included in this analysis.

The clinical pre- and postprocedural status of each patient was evaluated according to the modified Rankin Scale. Before the procedure, 88 patients were asymptomatic, $8(6.6 \%)$ had an mRS score of 1 or 2 caused by a symptomatic target aneurysm, and 25 (20.7\%) had an mRS score of $\geq 1$ unrelated to the target aneurysm.

Of the 130 aneurysms treated, the p64 procedure was the first treatment in 107 aneurysms, whereas 23 aneurysms were remnants or recurrences after previous coil occlusion $(n=20)$ or microsurgical clipping $(n=3)$. Previous rupture was confirmed for 6 aneurysms. The locations of the 130 treated aneurysms were as follows: ICA, cavernous $(n=8)$; ICA, paraoph-

AJNR Am J Neuroradiol 36:2082-89 Nov 2015 www.ajnr.org 
thalmic $(n=32)$; ICA, superior hypophyseal artery $(n=25)$; ICA, paraclinoid $(n=10)$; ICA, posterior communicating artery $(n=18)$; ICA, anterior choroidal artery $(n=10)$; anterior cerebral artery $(n=10)$; MCA $(n=4)$; vertebral artery/V4 $(n=4)$; posterior inferior cerebellar artery $(n=1)$; basilar artery $(n=4)$; superior cerebellar artery $(n=2)$; and posterior cerebral artery $(n=2)$.

The aneurysm fundus size was determined as the diameter of the perfused part of the aneurysm at the time of p64 implantation. The median size of the 130 treated aneurysms was $3 \mathrm{~mm}$ for the neck (range, $1-11 \mathrm{~mm}$ ) and $4 \mathrm{~mm}$ for the fundus (range, 1-20 $\mathrm{mm})$.

\section{Endovascular Procedure}

All procedures were performed in a single center with the patient under general anesthesia on biplane Axiom Artis DSA units (Siemens, Erlangen, Germany).

Either a $6 \mathrm{~F}$ guide catheter alone or a triaxial access was used. A 0.027 -inch-inner-diameter microcatheter was placed in a straight vessel segment of the parent artery distal to the aneurysm neck by using a 0.014 - or 0.016 -inch microguidewire.

After a suitable working projection was identified, the tar-

Table 1: Inclusion and exclusion criteria for the presented series of patients treated with p64

Criteria for the p64 Treatment and Analysis in this Series
Inclusion
Intracranial saccular sidewall aneurysm as treatment target
Aneurysms unruptured or at least not in the acute phase after rupture
Extra- or intradural symptomatic aneurysm
Asymptomatic intradural aneurysms, thus carrying a potential risk of intracranial rupture
Anticipated difficulty of coil or clip treatment (eg, complex aneurysm morphology, wide
neck, dome-to-neck ratio of $<1.2$, small size, difficult surgical access)
No previous treatment or any previous treatment directed at the aneurysm sac without
complete occlusion of the aneurysm from circulation
No previous treatment to the parent vessel
An ability and willingness of the patient to take the necessary medication for midterm
dual platelet function inhibition
Exclusion
Bifurcation-type aneurysm or fusiform vessel dilation as a treatment target
Implants other than p64 used
Aneurysm rupture $\leq 30$ days prior to the p64 treatment
Extradural asymptomatic aneurysm
Anticipated ease or sufficiency of clip or coil treatment
Previous implantation of stents or flow diverters to the target-vessel segment
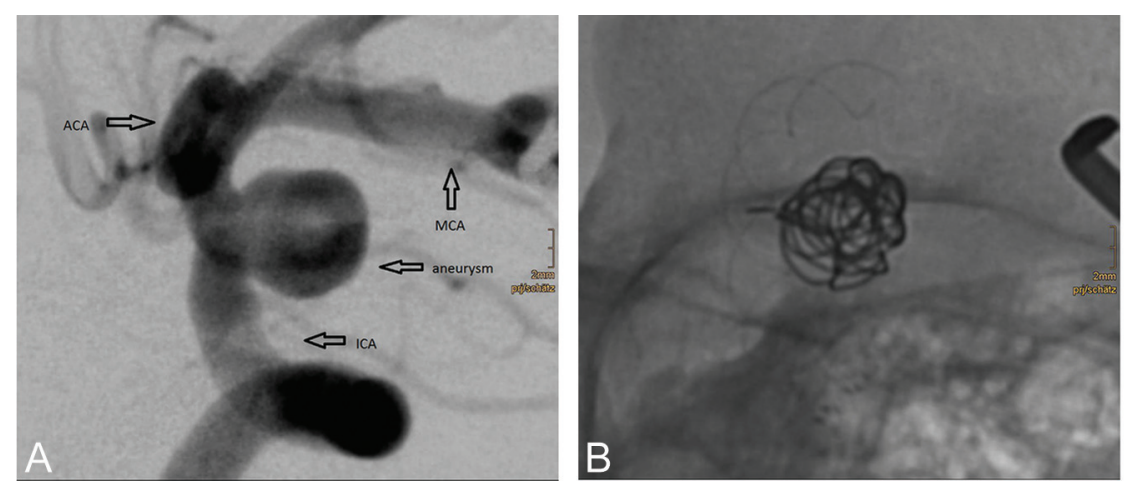

get-vessel diameter was measured, followed by device selection. The device matched the diameter of the healthy landing zone. Slight undersizing was possible because the diameter of the unconstrained implant is approximately $0.3 \mathrm{~mm}$ larger than the nominal diameter. This undersizing results in a denser coverage and shortening of the implant by a median length of $55 \%$ and was avoided if side branches were covered. Moderate oversizing was possible and resulted in a less attenuated coverage. The device was then prepared by pushing it out of and retracting it into the sheath, while submersed in saline. It was then advanced to the desired position via the microcatheter. Deployment was a combination of a slow withdrawal of the microcatheter, with a continuous counterpressure on the delivery wire. After the distal end of the device was anchored in the target vessel, the microcatheter was no longer pulled back. Pushing the delivery wire of the p64 resulted in a progressive deployment of the device with a passive proximal movement of the microcatheter. Once the proximal markers of the device were uncovered from the microcatheter, a single $\mathrm{x}$-ray image or a flat panel CT was performed to confirm the complete opening of the device. In case of incomplete opening, resheathing and redeployment resulted in better device expansion. The fully-opened device was mechanically detached as previously described. If the wall apposition of the p64, once detached, was not satisfactory, a compliant balloon was inserted and gently inflated.

In this series, coil occlusion and p64 deployment in 1 session were performed in $19(14.6 \%)$ aneurysms (Fig 3). The median fundus diameter of those partially coiled aneurysms was $8 \mathrm{~mm}$ (range, 4-20 mm); the median neck diameter was $5 \mathrm{~mm}$ (range, $2-8 \mathrm{~mm}$ ).

In 13 selected aneurysms (10\%), we implanted multiple p64s, concluding that 1 device might be insufficient (2 p64s, $n=9 ; 3$ p64s, $n=3$; 4 p64s, $n=1$ ). The decision concerning additional coil occlusion or the deployment of multiple p64 devices was at the operator's discretion.

FIG 3. De novo unruptured paraclinoid aneurysm $(7 \times 5 \mathrm{~mm})$ in a 63-year-old woman with a history of 2 spontaneous SAHs from 2 MCA bifurcation aneurysms, which had been clipped. The paraclinoid aneurysm was not considered ideal for coil occlusion alone ( $A$ ), and the patient was reluctant to undergo surgery again. A single Morpheus $7 \times 21 \mathrm{~cm} 3 \mathrm{D}$ coil (Medtronic, Dublin, Ireland) was inserted into the aneurysm. The aneurysm neck was then covered by a $4 \times$ $18 \mathrm{~mm}$ p64 (B). DSA follow-up 93 days later reveals complete occlusion of the aneurysm (C). 

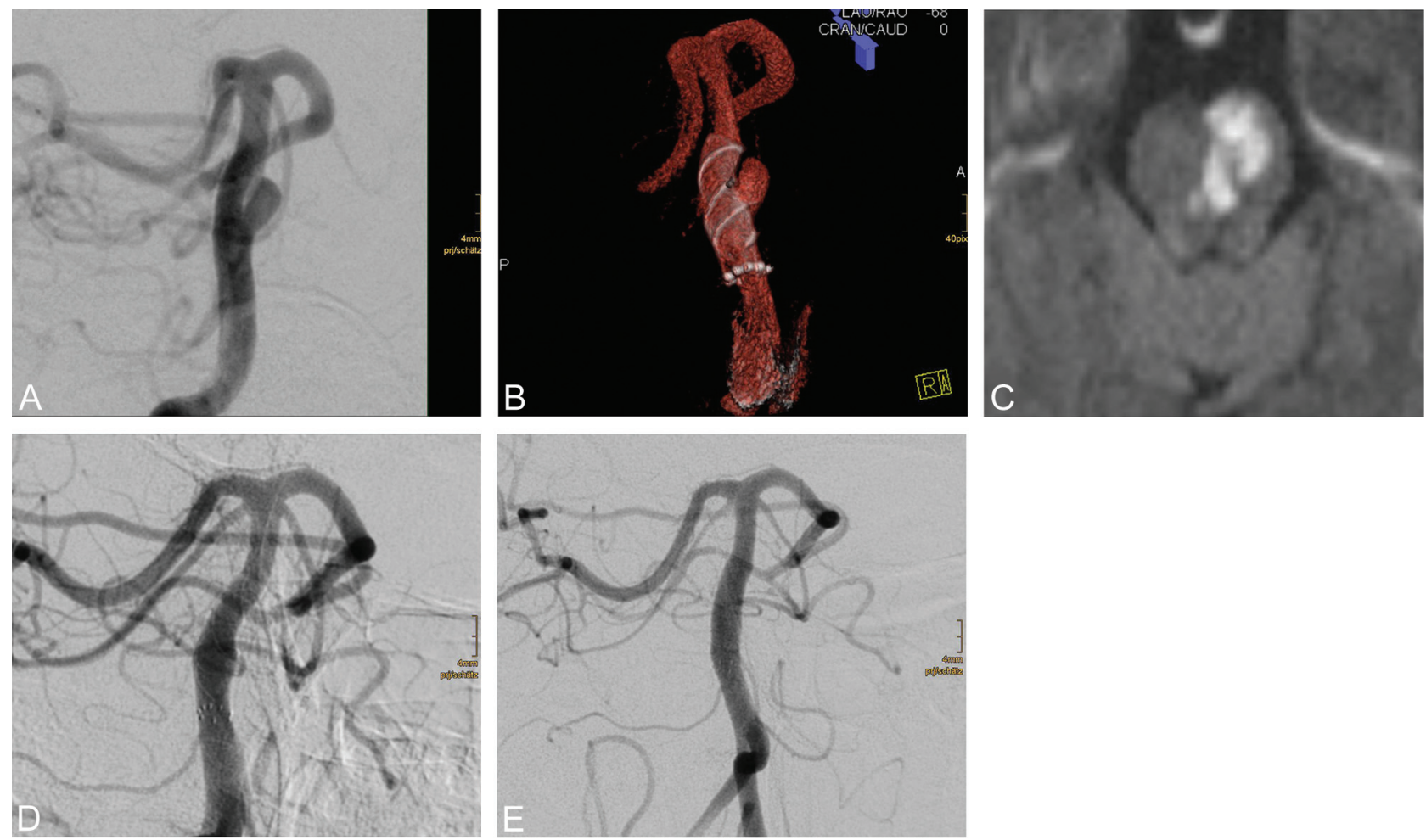

FIG 4. A small saccular aneurysm of the basilar trunk $(4 \times 3 \mathrm{~mm})(A)$ in a 56-year-old woman. Both surgery and coil occlusion were not considered suitable treatment options. A $4.5 \times 15 \mathrm{~mm}$ p64 was deployed in the basilar artery with complete coverage of the aneurysm (B). Although the procedure was well-tolerated, the patient developed a hemiparesis and dysarthria (mRS3) 26 days later. MR imaging shows an ischemic pontine lesion (C); a Multiplate test (not shown) confirmed insufficient platelet function inhibition. Antiaggregation was switched to ticagrelor, and the patient subsequently recovered (mRS 1). Follow-up DSA after 28 days $(D)$ and after 421 days $(E)$ shows complete occlusion of the aneurysm.

\section{Antiplatelet and Anticoagulation Regimen}

Patients received a loading dose of $600 \mathrm{mg}$ clopidogrel and $500 \mathrm{mg}$ acetylsalicylic acid at least 1 day before the procedure and were then maintained on dual antiplatelet medication of $75 \mathrm{mg}$ clopidogrel and $100 \mathrm{mg}$ acetylsalicylic acid daily. Platelet aggregation inhibition was tested before the procedure and occasionally thereafter with the impedance aggregometry (Multiplate; Roche, Basel, Switzerland) in all patients. Patients with clopidogrel resistance were switched to ticagrelor ( $2 \times 90 \mathrm{mg}$ daily) (Fig 4). ${ }^{8,9}$ Dual antiplatelet aggregation was continued for 1 year and was thereafter reduced to a monomedication of acetylsalicylic acid. The periprocedural medication included systemic heparinization.

\section{Follow-Up Schedule}

Patients were scheduled for clinical and angiographic follow-up examinations after treatment as follows:

- Early follow-up (3 months \pm 4 weeks)

- Intermediate follow-up (5-12 months)

- Late follow-up (>12 months).

A neurologist, a neurosurgeon, or a certified stroke nurse performed the clinical assessment by using the mRS. Clinical follow-up examinations compared the pretreatment and posttreatment clinical conditions of the patients.

Angiographic results were graded as follows: 1) complete occlusion, 2) neck remnant, 3) incomplete occlusion with residual sac filling, and 4) unchanged aneurysm perfusion. ${ }^{10}$

In-stent stenosis was graded as the following: 1, absent; 2,
$<50 \%$ lumen loss; 3, 50\%-75\% lumen loss; 4, >75\% lumen loss. If in-stent stenosis was observed, the lumen loss (LL) on subsequent follow-up DSA examinations was separately qualified as unchanged, decreased, or increased.

\section{Retreatment}

Retreatment was considered after follow-up angiographies revealed an unchanged, incomplete aneurysm occlusion or device migration.

\section{RESULTS}

\section{Procedural Technical Aspects and Difficulties}

Insertion, deployment, and detachment of the p64 was possible as intended in $127 / 130$ procedures $(97.7 \%)$. Excessive friction was encountered in 2 aneurysms. In 1 case, a remnant of a previously coiled pericallosal artery could not be reached due to friction of the p64 at the level of the Al segment. The aneurysm was treated in the same session with a PED with difficulty. In the second case, the target was an A1/A2 aneurysm. Serious friction was encountered during the attempted p64 insertion. In 2 subsequent sessions, an Enterprise self-expanding stent (Codman \& Shurtleff, Raynham, Massachusetts) was deployed, followed by the insertion of a p64 inside this stent. Follow-up DSA confirmed the occlusion of the aneurysm. In patient 3 , a basilar trunk aneurysm was reached and covered by a p64. The p64 was, however, withdrawn because a noncollateralized posterior cerebral artery would have been covered. The aneurysm was treated by Solitaire-assisted (Covidien) coil occlusion. In a subsequent session, a recurrence of this aneu- 
Table 2: Breakdown of aneurysm occlusion rate and complication incidence according to the size of the aneurysm fundus

\begin{tabular}{|c|c|c|c|c|}
\hline & \multicolumn{4}{|c|}{ Fundus Diameter } \\
\hline & $1-3 \mathrm{~mm}(n=59)$ & $4-6 \mathrm{~mm}(n=42)$ & 7-9 mm (n = 15) & $>10 \mathrm{~mm}(n=14)$ \\
\hline Early follow-up & $55 / 59(93 \%)$ & $42 / 42(100 \%)$ & $15 / 15(100 \%)$ & $12 / 14(85.7 \%)$ \\
\hline Complete occlusion & $31(56.4 \%)$ & $28(66.7 \%)$ & $9(60 \%)$ & $4(33.3 \%)$ \\
\hline Neck remnant & $13(23.6 \%)$ & $4(9.5 \%)$ & $2(13 \%)$ & $7(58.3 \%)$ \\
\hline Sac remnant & $7(12.7 \%)$ & 5 (11.9\%) & $3(20 \%)$ & $1(8.3 \%)$ \\
\hline Unchanged & $4(7.3 \%)$ & $4(9.5 \%)$ & $1(6.7 \%)$ & 0 \\
\hline Midterm follow-up & $46 / 59(78 \%)$ & $34 / 42(81 \%)$ & $11 / 15(73.3 \%)$ & 8/14 (57.1\%) \\
\hline Complete occlusion & $37(80.4 \%)$ & $26(76.5 \%)$ & $9(81.8 \%)$ & $4(50 \%)$ \\
\hline Neck remnant & $3(6.5)$ & $5(14.7 \%)$ & $2(18.2 \%)$ & $3(37.5 \%)$ \\
\hline Sac remnant & $4(8.7 \%)$ & $2(5.9 \%)$ & 0 & $1(12.5 \%)$ \\
\hline Unchanged & $2(4.3 \%)$ & $1(2.9 \%)$ & 0 & 0 \\
\hline \multirow[t]{3}{*}{ Complications } & 1 Pontine ischemia & 1 Pontine ischemia & $1 \mathrm{TIA}$ & 1 Death \\
\hline & 1CN VI palsy & $1 \mathrm{TIA}$ & 1 Asymptomatic thrombosis & 1 Pulmonary artery occlusion \\
\hline & 1 Asymptomatic thrombosis & & & \\
\hline
\end{tabular}

Note:-CN indicates cranial nerve.

rysm was treated with a p64. Follow-up DSA confirmed the complete occlusion of the aneurysm. Balloon expansion of the p64 after deployment was performed in 5 patients and was successful in all attempted cases.

\section{Early Peri- and Postprocedural Complications}

The peri- and postprocedural phases (24 hours) were clinically uneventful in $127 / 130$ procedures $(97.7 \%)$. One patient had a pulmonary artery embolism, and 2 patients had minor ischemic lesions with transient neurologic deficits.

The subacute phase (day 2 through 30 ) was within normal limits in $128 / 130$ procedures $(98.5 \%)$, and 2 patients experienced a pontine ischemic infarct. The clinical outcome of these patients was equivalent to $\mathrm{mRS} 1(n=1)$ as opposed to $\mathrm{mRS} 0$ before the treatment. In 1 of these 2 patients, dual platelet inhibition was interrupted against medical advice.

\section{Delayed Complications}

Beyond 30 days, 1 patient died from pneumonia, which was indirectly related to the endovascular treatment. This patient had received steroids for severe cranial nerve palsy due to the mass effect of a large ICA aneurysm, which had been treated by coil occlusion and a different flow diverter. In 2 patients, asymptomatic thrombus formation after cessation of dual antiplatelet aggregation was observed. In 1 patient, an unrelated cranial nerve VI palsy occurred 10 months posttreatment.

In the entire series, $6 / 121$ patients (5\%) experienced a transient morbidity ( 1 pulmonary artery embolism, 2 postprocedural TIAs, 1 late transient cranial nerve palsy, 2 asymptomatic thromboses requiring hospitalization). Two of 121 patients $(1.7 \%)$ developed a permanent morbidity, and $1 / 121$ patients $(0.8 \%)$ died. Neither aneurysm rupture nor parenchymal hemorrhage was observed after p64 treatment.

\section{Angiographic Follow-Up}

An early follow-up DSA examination was performed after a median of 92 days in 123/130 aneurysms (94.6\%). The remaining 7 aneurysms included 3 failed treatment attempts, 1 patient who had died, 2 patients who refused follow-up examinations, and 1 pending DSA. Complete aneurysm occlusion was confirmed in $72(58.5 \%)$, and a neck remnant, in $26(21.1 \%)$ an- eurysms. For 16 (13\%) aneurysms, a sac remnant was found, and $9(7.3 \%)$ aneurysms were unchanged.

An intermediate follow-up DSA was available in 93/106 eligible aneurysms (87.7\%) treated until May 2014 after a median of 279 days, showing a complete aneurysm occlusion in 74 $(79.6 \%)$ and a neck remnant in $13(14 \%)$ aneurysms. For 4 (4.3\%) aneurysms, a sac remnant was found, and $2(2.2 \%)$ aneurysms were unchanged. The missing 13 aneurysms include 3 failed treatment attempts, 1 patient who died, 1 aneurysm that was retreated, 5 patients refusing follow-up DSA, and 3 pending examinations.

A late follow-up DSA (after 496 days, median) has been performed already in 35 aneurysms, revealing complete occlusion in $30(85.7 \%)$ and neck remnant in $5(14.3 \%)$ aneurysms, respectively.

Asymptomatic usually transient in-stent stenosis was identified in 24/123 (19.5\%) patients during the early follow-up DSA (LL, $<50 \%, n=11$; LL, $50 \%-75 \%, n=11$; LL, $>75 \%, n=2)$. The midterm follow-up DSA revealed in-stent stenosis in 11/93 (11.8\%) patients (LL, $<50 \%, n=9$; LL, 50\%-75\%, $n=2 ; \mathrm{LL},>75 \%, n=0$ ). The late follow-up DSA showed no in-stent stenosis with a LL of $\geq 50 \%$.

Three patients underwent uneventful balloon angioplasty for asymptomatic in-stent stenoses. In 15 patients, in-stent stenosis significantly improved after the first follow-up DSA without any additional treatment.

Retreatment of the target lesion was performed in 5 patients. In 3 of these patients, the effect of the first implant was considered insufficient. In 2 patients, the first p64 had shortened from distal to proximal, leaving the aneurysm neck uncovered. All 5 patients were retreated by deploying another p64. All retreatments were performed and tolerated without any adverse events.

\section{Clinical Follow-Up}

The mRS score improved in 8 patients $(6.6 \%)$ and remained unchanged in 110 patients $(90.9 \%)$. Clinical deterioration was observed in 3 patients $(2.5 \%)$. The rates of transient morbidity, permanent morbidity, and mortality in this series are $5 \%$, $1.7 \%$, and $0.8 \%$, respectively. 
Table 2 shows a breakdown of aneurysm occlusion rates and complication incidences according to the size of the aneurysm fundus. The cumulative numbers for complete occlusion and neck remnants are given. All complications are listed.

\section{DISCUSSION}

This retrospective study presents our experience in the treatment of intracranial saccular sidewall aneurysms with the p64, a mechanically detachable flow diverter. The technical success rate, clinical outcome, and angiographic results are in line with the findings of comparable series. ${ }^{1,3-5,11-13}$ The device itself is being developed further, similar to modifications to the PED and NeuroEndoGraft.

\section{Flow Diversion}

Endovascular treatment of wide-neck and very small aneurysms can be challenging. The concept of redirecting the blood flow away from the aneurysm along the parent artery with subsequent intra-aneurysmal thrombosis resulted in the development of flow diverters. This effort led to numerous in vitro hemodynamic studies that identified porosity (defined as the percentage of open surface area relative to the entire surface area of the device) and pore and filament size as essential factors. ${ }^{14,15}$ A porosity of approximately $70 \%$ was shown to be ideal for adequate hemodynamic efficiency without a relevant loss of flexibility and negligible effects on the parent artery and the side branches. Most of the currently available flow diverters, as well as the p64, are designed with a porosity of approximately $70 \%$ at nominal diameters. Compared with 48 wires for PED and Silk+, the p64 is a braid of 64 nitinol wires, which results in denser coverage across the aneurysm neck. It features a controlled mechanical detachment mechanism, which allows repositioning or withdrawal of the device even after complete deployment.

Issues after flow-diverting procedures are frequently the result of malpositioning or incomplete opening of the implant. In their initial presentation of 63 aneurysms treated with the PED, Lylyk et $\mathrm{al}^{11}$ reported 2 cases with deployment issues. One was a dislocation of the proximal end of the device into the aneurysm, which was repositioned with a retrieval device. In the other case, the distal tip of the delivery wire fractured. These findings are similar to our own experience with the PED. ${ }^{1}$

The option to withdraw and redeploy the p64 helped avoid several of the previously encountered issues.

Berge et $\mathrm{al}^{3}$ reported 7 malpositioned Silk flow diverters in their series of 77 aneurysms. Incomplete opening of the central part of the device with poor vessel wall apposition seems to occur more frequently in curved and elongated vessels and when a longer device is used and is associated with a higher rate of in-stent thrombosis. ${ }^{16}$ The use of a compliant balloon to achieve complete wall apposition is technically straightforward and reduces the incidence of endoleaks. In our series, there were occasional instances of incomplete proximal opening of the p64 that were corrected by manipulation with the microguidewire or the microcatheter. Balloon angioplasty was performed in 5 procedures $(3.8 \%)$. As a rule of thumb, shorter devices deploy better, show less twisting, and allow more consistent wall apposition. Mechanical detachment with the possibility of optimizing the final position of the device plays a key role in the abatement of device-related issues and might significantly improve long-term clinical and angiographic results.

Side branch occlusions may be avoided by using fewer devices. Kulcsár et $\mathrm{al}^{17}$ discussed 2 potential mechanisms leading to side branch occlusions: mechanical blockade and a narrowing of the neck or conduction of embolic material from the surface of the flow diverter. They encountered 2 cases of side branch occlusions in their series of 12 basilar artery aneurysms treated with the Silk. In one of these patients, the flow diverter was placed in a previously implanted stent. ${ }^{17}$ Other studies showed that side branch occlusions do not necessarily result in symptomatic ischemia in patients with adequate collateral circulation. Szikora et al ${ }^{18}$ reported 19 wide-neck aneurysms treated with the PED and detected 3 clinically silent occlusions of the ophthalmic artery. In 2 patients, 3 and 4 PEDs, respectively, had been deployed. ${ }^{18}$

The fact that symptomatic side branch occlusion after flow diverter treatment occurs more frequently in the posterior circulation may be due to the vessel anatomy with pontine arteries originating from the basilar trunk. This is re-emphasized by our observation of 2 perforator strokes related to p64 implantation in the basilar artery. In the basilar artery and in the proximal MCA, undersizing the p64 should be avoided because increased coverage of perforating vessels may result. Slight oversizing is associated with less coverage, but increased chronic outward force might be a stimulus for in-stent stenosis, which might cause delayed perforator occlusion.

In our practice, the decision to implant multiple devices was at the operator's discretion. Arguments in favor of $>1$ device included unchanged aneurysm perfusion after implantation of the first device and segmental vessel disease with very wide-neck aneurysms. In this select series, $>1$ p 64 was used in 13/127 successful procedures $(10.2 \%)$, with an average of 1.1 p64 per procedure. This value is significantly lower than the respective number in our PED series, with $>1$ PED in 67 of 101 treated cases, ${ }^{1}$ with an average of 3.2 PEDs per procedure, yet our initial follow-up results are comparable with or better than those published for the PED and Silk, respectively. The currently available data, however, do not yet allow a comparison of the aneurysm occlusion rate of the p64 versus other devices. A single-device strategy might result in a lower procedural complication rate, but there is nothing magical about a "one and done" approach to treatment with a flow diverter. Complete occlusion of an aneurysm occurs when braided filaments of the flow diverter are covered by neointima across the neck. In very wide, highly concave necks or fusiform segments, the amount of braid material available for neointima to grow on may not be sufficient to enable complete endothelial growth. A continuous blood/thrombus interface across the neck keeps the aneurysm alive. For rapid neointima coverage at the neck, multiple devices may be the only solution. While economic factors may impact operators' decisions, nevertheless, patients with such aneurysms must be adequately treated. All these theoretic arguments await reconsideration in significantly larger series and eventually in comparative trials.

The experience of our institution may be 1 reason for the low 
rate of multiple-device complications in this series. By the time we used the first p64, $>200$ patients had already been treated with the PED.

\section{Device Selection of the $p 64$}

Chalouhi et $\mathrm{al}^{19}$ presented 5 selected patients with spontaneous delayed migration or shortening of the PED from a series of 155 patients. Two of these observations resulted in serious clinical issues ( 1 fatal SAH and 1 permanent disability due to a MCA occlusion). Correct device selection, based on precise measurements of healthy landing zones, is critical to proper deployment and treatment. A correlation between the diameter of the target vessel and the resulting length of the device is provided for each p64. A $4.5 \times 21 \mathrm{~mm}$ device at minimum will expand to a diameter of $4.5 \mathrm{~mm}$ and a length of $21 \mathrm{~mm}$ but will lengthen to $28 \mathrm{~mm}$ when deployed into a $4 \mathrm{~mm}$ vessel, which results in a decrease of surface coverage. Moderate oversizing of the implant may be used intentionally to increase the porosity (eg, in perforator-rich territories). We observed delayed shortening of the p64 in 2 patients, requiring a second treatment.

\section{Hemorrhagic Complications}

Intracranial hemorrhages after flow-diversion procedures raised a major concern. This phenomenon is not common after endovascular coiling or microsurgical clipping.

Several reports of delayed aneurysm rupture after flow diversion have been published. The hemodynamic theory assumes a valve mechanism, induced by the flow diverter, which increases the wall stress to the aneurysm. The enzymatic theory, which appears more convincing, is based on the assumption that thrombus releases enzymes that cause lysis of the aneurysm wall. ${ }^{20}$

In a series of 295 intracranial aneurysms treated with PED or Silk in 25 Italian centers, the authors reported a high incidence of aneurysm ruptures after treatment of large and giant aneurysms, even though the flow diverters were used in combination with coils. The mortality in the subgroup of patients treated with additional coils was $35.7 \%$. These data suggest that the use of coils does not prevent delayed aneurysm ruptures. ${ }^{2}$ Kulcsár et $\mathrm{al}^{21}$ reported that very large aneurysms containing a heavy thrombus burden were most prone to delayed rupture. We have not observed any case of delayed aneurysm rupture in our p64 series so far. One possible explanation is that aneurysms with a fundus diameter of $\geq 10 \mathrm{~mm}$ were at least partially coiled before flow-diversion treatment.

Parenchymal hemorrhages distal to the target vessel, occurring several days after flow-diverter treatment, may have different underlying causes. The incidence of delayed parenchymal hemorrhages in the Retrospective Analysis of Delayed Aneurysm Ruptures after Flow Diversion study was $1.9 \%$. This retrospective multicenter study analyzed the incidence of hemorrhagic events after flow diversion. ${ }^{21}$

The underlying mechanism for remote parenchymal hemorrhages remains unclear. Periprocedural microguidewire perforations are an implausible explanation. Hemorrhagic transformation of clinically silent procedural embolic infarcts was proposed. ${ }^{1}$
$\mathrm{Hu}$ et $\mathrm{al}^{22}$ performed a histopathologic assessment of brain sections of 3 patients with fatal ipsilateral intracranial hemorrhages after uneventful treatment with the PED. They detected a nonbiologic material occluding the lumen of the vessels in the region of the hemorrhage. They suggested that a rise in venule pressure due to foreign body embolic occlusion was responsible for an increased microvascular permeability, resulting in the ipsilateral hemorrhagic infarction. The material was polyvinylpyrrolidone, which is part of the coating of many neurovascular devices. One explanation for the absence of delayed hemorrhages in the presented series might be found in the preparation of the p64 by complete submersion of the device in saline before the deployment to remove trapped air bubbles.

\section{CONCLUSIONS}

The p64 offers an effective treatment option for intracranial aneurysms with low complication rates, particularly due to the ability to reposition and/or remove the device in cases of poor deployment. It allows complete aneurysm occlusion within a few months, mostly achieved with a single device. Safety margins are within expected limits, with device-related complications being infrequent. A prospective study to confirm these findings is warranted.

Disclosures: Marta Aguilar-Pérez-UNRELATED: Consultancy: phenox. Wiebke Kurre-UNRELATED: Consultancy: phenox; Grants/Grants Pending: Covidien,* Comments: research grant for stroke research; Payment for Lectures (including service on Speakers Bureaus): phenox. Hans Henkes-RELATED: Consulting Fee or Honorarium: phenox, Comments: I have a consulting contract and a proctoring contract with phenox, which includes compensation on a fee-for-service basis; Support for Travel to Meetings for the Study or Other Purposes: phenox, Comments: compensation for expenses related to the participation in meetings with presentation of p64 data; Other: phenox, Comments: I am cofounder and shareholder of phenox; UNRELATED: Board Membership: phenox, Comments: Expenses related to board meetings are compensated; Grants/Grants Pending: Siemens, ${ }^{*}$ Covidien,* Comments: Siemens: DynaCT projects; Covidien: stroke research; Payment for Lectures (including service on Speakers Bureaus): ev3/Covidien, Comments: presentations related to the Pipeline flow diverter; Patents (planned, pending or issued): Dendron, phenox, Comments: participation in numerous patents filed by Dendron and phenox; Royalties: phenox; Stock/Stock Options: phenox; Travel/Accommodations/Meeting Expenses Unrelated to Activities Listed: AB Medica, ${ }^{*}$ Comments: compensation for physician training. *Money paid to the institution.

\section{REFERENCES}

1. Fischer S, Vajda Z, Aguilar Perez M, et al. Pipeline embolization device (PED) for neurovascular reconstruction: initial experience in the treatment of 101 intracranial aneurysms and dissections. Neuroradiology 2012;54:369-82

2. Briganti F, Napoli M, Tortora, et al. Italian multicenter experience with flow-diverter devices for intracranial unruptured aneurysm treatment with periprocedural complications: a retrospective data analysis. Neuroradiology 2012;54:1145-52

3. Berge J, Biondi A, Machi P, et al. Flow-diverter Silk stent for the treatment of intracranial aneurysms: 1-year follow-up in a multicenter study. AJNR Am J Neuroradiol 2012;33:1150-55

4. De Vries J, Boogaarts J, Van Norden A, et al. New generation of flow diverter (Surpass) for unruptured intracranial aneurysms: a prospective single-center study in 37 patients. Stroke 2013;44:1567-77

5. Becske T, Kallmes DF, Saatci I, et al. Pipeline for uncoilable or failed aneurysms: results from a multicenter clinical trial. Radiology 2013;267:858-68

6. Mallik AS, Nuss K, Kronen PW, et al. A new-generation, low-permeability flow diverting device for treatment of saccular aneurysms. Eur Radiol 2014;24:12-18

7. Anzai H, Falcone JL, Chopard B, et al. Optimization of strut place- 
ment in flow diverter stents for four different aneurysm configurations. J Biomech Eng 2014;136:061006

8. O I, M O, A AS, et al. Evaluation of aspirin and clopidogrel resistance in patients with acute coronary syndrome using Adenosine Diphosphate Test and Aspirin Test. Pak J Med Sci 2013;29:97-102

9. Del Castillo-Carnevali H, Barrios Alonso V, Zamorano Gomez JL. Antiplatelet therapy: resistance to traditional antiaggregation drugs and role of new antiplatelet agents [in Spanish]. Med Clin (Barc) 2014;143:222-29

10. Campi A, Ramzi N, Molyneux AJ, et al. Retreatment of ruptured cerebral aneurysms in patients randomized by coiling or clipping in the International Subarachnoid Aneurysm Trial (ISAT). Stroke 2007;38:1538-44

11. Lylyk P, Miranda C, Ceratto R, et al. Curative endovascular reconstruction of cerebral aneurysms with the Pipeline embolization device: the Buenos Aires experience. Neurosurgery 2009;64:632-42

12. D'Urso PI, Lanzino G, Cloft HJ, et al. Flow diversion for intracranial aneurysms: a review. Stroke 2011;42:2363-68

13. Zhou Y, Yang PF, Fang YB, et al. A novel flow-diverting device (Tubridge) for the treatment of 28 large or giant intracranial aneurysms: a single-center experience. AJNR Am J Neuroradiol 2014;35:2326-33

14. Augsburger L, Fahrhat M, Reymond P, et al. Effect of flow diverter porosity an intraaneurysmal blood flow. Klin Neuroradiol 2009;19:204-14

15. Seshadhri S, Janiga G, Beuing O, et al. Impact of stents and flow diverters on hemodynamics in idealized aneurysm models. $J$ Biomech Eng 2011;133:071005
16. Wagner A, Cortsen M, Hauerberg J, et al. Treatment of intracranial aneurysms: reconstruction of the parent artery with flow-diverting (Silk) stent. Neuroradiology 2012;54:709-18

17. Kulcsár Z, Ernemann U, Wetzel SG, et al. High-profile flow diverter (Silk) implantation in the basilar artery: efficacy in the treatment of aneurysms and the role of the perforators. Stroke 2010;41:1690-96

18. Szikora I, Berentei Z, Kulcsar Z, et al. Treatment of intracranial aneurysms by functional reconstruction of the parent artery: the Budapest experience with the Pipeline embolization device. AJNR Am J Neuroradiol 2010;31:1139-47

19. Chalouhi N, Tjoumakaris SI, Gonzalez LF, et al. Spontaneous delayed migration/shortening of the Pipeline embolization device: report of 5 cases. AJNR Am J Neuroradiol 2013;34:2326-30

20. Turowski B, Macht S, Kulcsàr Z, et al. Early fatal hemorrhage after endovascular cerebral aneurysm treatment with a flow diverter (SILK-Stent): do we need to rethink our concepts? Neuroradiology 2011;53:37-41

21. Kulcsàr Z, Szikora I. The ESMINT Retrospective Analysis of Delayed Aneurysm Ruptures after Flow Diversion (RADAR) study. The eJournal of the European Society of Minimally Invasive Neurological Therapy 2012;1244000088. http://www.ejmint.org/ sites/default/files/pdf/original_article_1244000088.pdf. Accessed October 29, 2012

22. Hu YC, Deshmukh VR, Albuquerque FC, et al. Histopathological assessment of fatal ipsilateral intraparenchymal hemorrhages after the treatment of supraclinoid aneurysms with the Pipeline embolization device. J Neurosurg 2014;120:365-74 